\title{
Formation of Core-Mantle Type Grains Consisting of a SiC Core and a Carbon Mantle in Circumstellar Envelopes of Carbon Stars
}

\section{TAKASHI KOZASA $^{1}$ and HISATO SOGAWA ${ }^{2}$}

${ }^{1}$ Department of Earth and Planetary Sciences, Kobe University, Japan

${ }^{2}$ Department of Physics, Kyoto University, Japan

We have proposed that the so-called $11.3 \mu \mathrm{m}$ feature observed towards carbon stars can be attributed to small spherical core-mantle type grains consisting of a $\mathrm{SiC}$ core and a carbon mantle, using the optical constants of SiC tabulated by Choyke \& Palik (1985, in Handbook of Optical Constants of Solids). Also we demonstrated qualitatively the possibility of formation of such a grain on the basis of a theory of nucleation and grain growth with a simple model of the circumstellar envelopes (Kozasa et al. 1996, $A \& A, 307$, 551). Radiative transfer calculations have shown that the proposed dust grains reasonably reproduce the observed spectral features if the volume fraction of the carbon mantle increases with increasing distance in the outflow. In order to realize the formation of the proposed core-mantle type grains quantitatively, we have investigated the formation of $\mathrm{SiC}$ and carbon grains as well as the accretion of a carbon mantle on pre-condensed $\mathrm{SiC}$ grains together with the gas flow caused by the radiation pressure forces acting on the newly formed grains in the circumstellar envelope. The result of calculations shows that, after the avalanche of formation of $\mathrm{SiC}$ grains, accretion of carbon mantles on $\mathrm{SiC}$ and formation of carbon grains proceed in competition with each other. The volume fraction of the carbon mantle as well as the amount of isolated carbon grains increases as the mass-loss rate of carbon stars increases. We discuss the dependence of the structure and size of dust grains formed in the circumstellar envelopes on the mass-loss rate of carbon stars. 